\title{
UNIVERSAL CURRENT-CONTROLLED CURRENT-MODE FILTER WITH THREE- INPUTS AND ONE-OUTPUT USING THE CURRENT CONTROLLED CONVEYOR
}

\author{
MUHAMMAD TAHER ABUELMA'ATTI* \\ and MUHAMMAD ALI AL-QAHTANI \\ King Fahd University of Petroleum and Minerals, Box 203, Dhahran 31261, \\ Saudi Arabia
}

(Received 18 November 1997; In final form. 23 January 1998)

\begin{abstract}
A new current-mode universal filter is proposed. The filter uses five current-controlled current-conveyors (CCCII) and can realise lowpass, highpass, bandpass, notch and allpass responses. The parameters $\omega_{o},\left(\omega_{o} / Q_{o}\right)$ can be controlled by adjusting the bias currents of the CCCIIs. The proposed circuit enjoys low sensitivities.
\end{abstract}

Keywords: Current conveyors; current-mode filters

\section{INTRODUCTION}

Universal active filters using the operational transconductance amplifier (OTA) have many advantages such as simplicity, integratability and programmability [1-5]. However, they have some problems with dynamic range and at high frequencies of operation. On the other hand, current-mode current-conveyor based filters can offer wider signal bandwidths, greater linearity and larger dynamic ranges of operation [6-18]. However, they lack programmability. While programmability can be achieved by combining current conveyors and OTAs [19], the recently introduced second-generation current-controlled conveyor (CCCII) [20] allows current conveyor

*Corresponding author. 
applications to be extended to the domain of electronically programmable functions. Figure 1(a) shows the electrical symbol of the $\mathrm{CCCII}+$. Using two CCCII + and two grounded capacitors, a voltage-mode bandpass filter was reported [20] and a current-mode bandpass filter was reported [21]. No attempt has been reported, so far, to present universal second-order current-mode filters using the
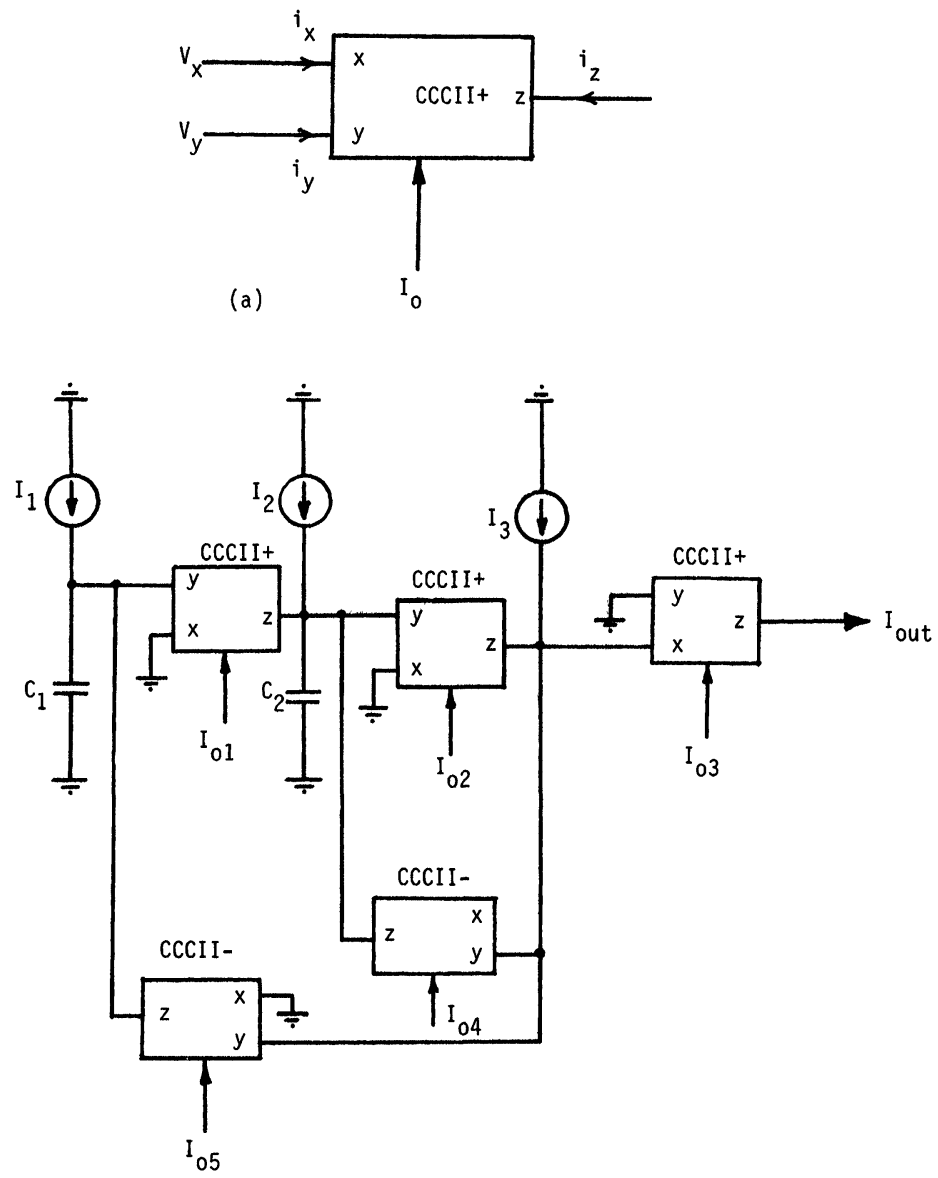

(b)

FIGURE 1 (a) Electrical symbol of the plus-type current-controlled current conveyor. (b) Universal current-controlled current-mode filter. 
$\mathrm{CCCII} \pm$ and grounded capacitors only. It is the purpose of this paper to present such a configuration.

\section{PROPOSED CIRCUIT}

The proposed circuit is shown in Figure 1(b). Using standard notations, the CCCII \pm can be characterised by $i_{y}=0, V_{x}=V_{y}+R_{x} i_{x}$ and $i_{z}= \pm i_{x}$, where $R_{x}=V_{T} / 2 I_{o}, V_{T}$ is the thermal voltage and $I_{o}$ is the bias current of the CCCII. Routine analysis yields the current transfer function which can be expressed by

$$
I_{\mathrm{out}}=-\frac{s^{2} I_{3}+s\left(1 / C_{2} R_{x 2}\right) I_{2}+\left(1 / C_{1} C_{2} R_{x 1} R_{x 2}\right) I_{1}}{s^{2}+s\left(R_{x 3} / C_{2} R_{x 2} R_{x 4}\right)+\left(\left(R_{x 3} / R_{x 5}\right) / C_{1} C_{2} R_{x 1} R_{x 2}\right)}
$$

Now, a current-mode bandpass filter is obtained if we choose $I_{1}=I_{3}=0$, thus

$$
\frac{I_{\mathrm{BP}}}{I_{2}}=-\frac{s / C_{2} R_{x 2}}{s^{2}+s\left(R_{x 3} / C_{2} R_{x 2} R_{x 4}\right)+\left(\left(R_{x 3} / R_{x 5}\right) / C_{1} C_{2} R_{x 1} R_{x 2}\right)}
$$

A current-mode lowpass filter is obtained if we choose $I_{2}=I_{3}=0$, thus

$$
\frac{I_{\mathrm{LP}}}{I_{1}}=-\frac{\left(1 / C_{1} C_{2} R_{x 1} R_{x 2}\right)}{s^{2}+s\left(R_{x 3} / C_{2} R_{x 2} R_{x 4}\right)+\left(\left(R_{x 3} / R_{x 5}\right) / C_{1} C_{2} R_{x 1} R_{x 2}\right)}
$$

A current-mode highpass filter is obtained if we choose $I_{1}=I_{2}=0$, thus

$$
\frac{I_{\mathrm{HP}}}{I_{3}}=-\frac{s^{2}}{s^{2}+s\left(R_{x 3} / C_{2} R_{x 2} R_{x 4}\right)+\left(\left(R_{x 3} / R_{x 5}\right) / C_{1} C_{2} R_{x 1} R_{x 2}\right)}
$$

A current-mode notch (bandreject) filter is obtained if we choose $I_{2}=0, I_{1}=I_{3}=I_{i}$ and $R_{x 3}=R_{x 5}$, thus

$$
\frac{I_{\mathrm{NOTCH}}}{I_{i}}=-\frac{s^{2}+\left(1 / C_{1} C_{2} R_{x 1} R_{x 2}\right)}{s^{2}+s\left(R_{x 3} / C_{2} R_{x 2} R_{x 4}\right)+\left(\left(R_{x 3} / R_{x 5}\right) / C_{1} C_{2} R_{x 1} R_{x 2}\right)}
$$


And a current-mode allpass filter is obtained if we choose $I_{1}=I_{3}=-I_{2}=I_{i}$ and $R_{x 3}=R_{x 4}=R_{x 5}$, thus

$$
\frac{I_{\mathrm{AP}}}{I_{i}}=-\frac{s^{2}-s\left(1 / C_{2} R_{x 2}\right)+\left(1 / C_{1} C_{2} R_{x 1} R_{x 2}\right)}{s^{2}+s\left(1 / C_{2} R_{x 2}\right)+\left(1 / C_{1} C_{2} R_{x 1} R_{x 2}\right)}
$$

From Eqs. $(2-6)$ the parameters $\omega_{o}$ and $\omega_{o} / Q_{o}$ can be expressed as

$$
\omega_{o}^{2}=\frac{R_{x 3} / R_{x 5}}{C_{1} C_{2} R_{x 1} R_{x 2}}
$$

and

$$
\frac{\omega_{o}}{Q_{o}}=\frac{R_{x 3}}{C_{2} R_{x 2} R_{x 4}}
$$

From Eqs. $(2-4)$ it can be seen that the high frequency gain of the highpass filter is equal to unity, the dc gain of the lowpass filter is equal to

$$
G_{\mathrm{LP}}=\frac{R_{x 5}}{R_{x 3}}
$$

and the bandpass gain at $\omega_{o}$ is equal to

$$
G_{\mathrm{BP}}=\frac{R_{x 4}}{R_{x 3}}
$$

From Eqs. $(7-10)$ it can be seen that the paramter $\omega_{o}$ can be adjusted by controlling the resistance $R_{x 1}$, that is the bias current $I_{o 1}$, without disturbing the parameters $\omega_{o} / Q_{o}$, the lowpass gain $G_{\mathrm{LP}}$ and the bandpass gain, $G_{\mathrm{BP}}$. Moreover, the parameter $\omega_{o} / Q_{o}$ can be adjusted by controlling the resistance $R_{x 2}$, that is the bias current $I_{o 2}$, without disturbing the parameter $\omega_{o}$, the lowpass gain, $G_{\mathrm{LP}}$ and the bandpass gain $G_{\mathrm{BP}}$. Furthermore, the bandpass gain can be adjusted by controlling the resistance $R_{x 4}$, that is the bias current $I_{o 4}$, without disturbing the parameter $\omega_{o}$. However, this will disturb the parameter $\omega_{o} / Q_{o}$. Finally, the lowpass gain can be adjusted by controlling the resistance $R_{x 5}$, that is the bias current $I_{o 5}$, without disturbing the 


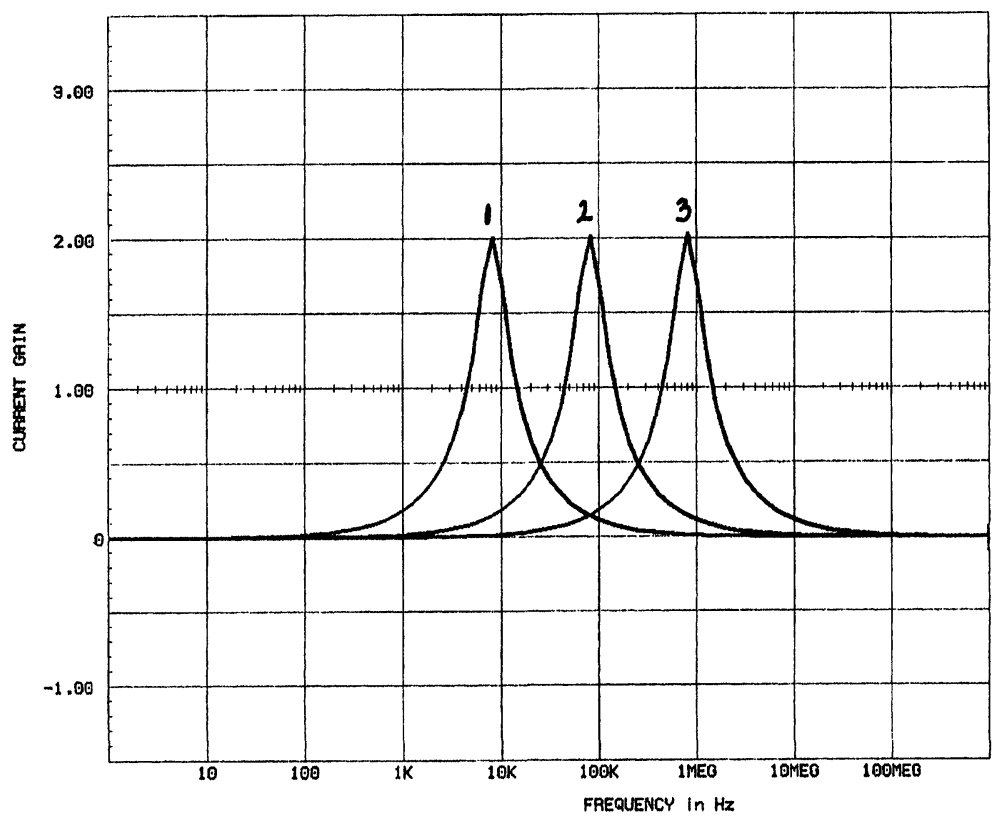

(a)
1: $I_{01}=1 u A$
2: $I_{01}=10 \mathrm{uA}$
3: $I_{01}=100 \mathrm{uA}$

FIGURE 2 Simulated gain-frequency characteristics of the (a) BPF obtained from the circuit of Figure 1 with $C_{1}=C_{2}=1000 \mathrm{pF}, I_{o 1}=I_{o 2}=1 \mu \mathrm{A}, 10 \mu \mathrm{A}, 100 \mu \mathrm{A}$, $I_{o 3}=100 \mu \mathrm{A}, I_{o 4}=I_{o 5}=50 \mu \mathrm{A}, I_{1}=I_{3}=0, I_{2}=1 \mu \mathrm{A}$ (b) LPF obtained from the circuit of Figure 1 with $C_{1}=C_{2}=1000 \mathrm{pF}, I_{o 1}=I_{o 2}=1 \mu \mathrm{A}, 10 \mu \mathrm{A}, 100 \mu \mathrm{A}$, $I_{o 3}=I_{o 4}=100 \mu \mathrm{A}, I_{o 5}=50 \mu \mathrm{A}, I_{2}=I_{3}=0, I_{1}=1 \mu \mathrm{A}$ (c) $\mathrm{HPF}$ obtained from the circuit of Figure 1 with $C_{1}=C_{2}=1000 \mathrm{pF}, I_{o 1}=I_{o 2}=1 \mu \mathrm{A}, 10 \mu \mathrm{A}, 100 \mu \mathrm{A}$, $I_{o 3}=100 \mu \mathrm{A}, I_{o 4}=100 \mu \mathrm{A}, I_{o 5}=50 \mu \mathrm{A}, I_{1}=I_{2}=0, I_{3}=1 \mu \mathrm{A}$.

parameter $\omega_{o} / Q_{o}$. However, this will disturb the parameter $\omega_{o}$. A possible strategy for adjusting the parameters $\omega_{o}, \omega_{o} / Q_{o}$ and the bandpass or the lowpass gains is therefore as follows: first the resistance $R_{x 4}$ or $R_{x 5}$, that is the bias current $I_{o 4}$ or $I_{o 5}$, is adjusted to control the bandpass gain $G_{\mathrm{BP}}$ or the lowpass gain $G_{\mathrm{LP}}$, then the bias current $I_{o 1}$ is adjusted to control the parameter $\omega_{o}$ and finally the bias current $I_{o 2}$ is adjusted to control the bandpass gain. 


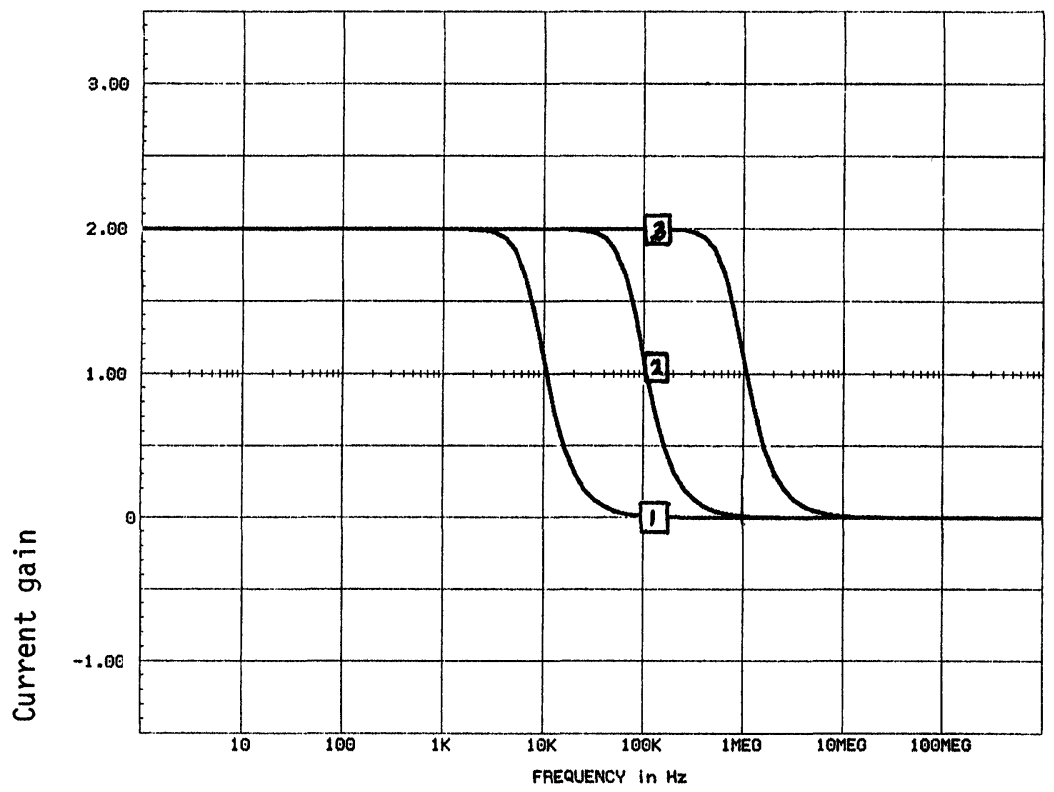

(b)
$1: I_{01}=1 \mathrm{uA}$
2: $I_{01}=10 \mathrm{UA}$
3: $I_{01}=100 \mathrm{uA}$

FIGURE 2 (Continued).

From Eqs. (7 and 8) it is easy to show that the sensitivities of the parameters $\omega_{o}$ and $Q_{o}$ are

$$
S_{R_{x 1}}^{\omega_{o}}=S_{R_{x 2}}^{\omega_{o}}=S_{R_{x 5}}^{\omega_{o}}=S_{C_{1}}^{\omega_{o}}=S_{C_{2}}^{\omega_{o}}=-S_{R_{x 3}}^{\omega_{o}}=-\frac{1}{2}
$$

and

$$
S_{R_{x 2}}^{\omega_{o} / Q_{o}}=-S_{R_{x 3}}^{\omega_{o} / Q_{o}}=S_{R_{x 4}}^{\omega_{o} / Q_{o}}=S_{C_{2}}^{\omega_{o} / Q_{o}}=-1
$$

all of which are small. 


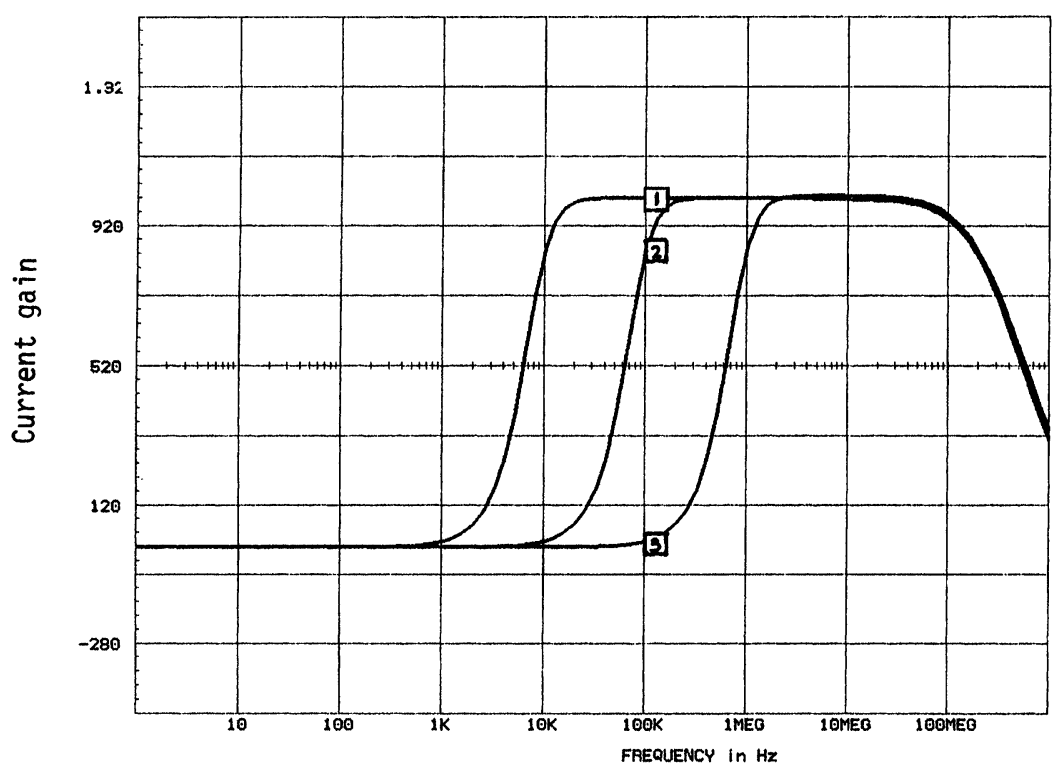

(c)
$1: I_{01}=1 \mathrm{uA}$
2: $I_{01}=10 \mathrm{uA}$
3: $I_{01}=100 \mathrm{UA}$

FIGURE 2 (Continued).

\section{SIMULATION RESULTS}

The universal filter circuit of Figure 1(b) has been simulated using the ICAPS circuit simulation program. The CCCII \pm have been simulated using the schematic implementation of [20] with dc supply voltage $= \pm 2.5 \mathrm{~V}$. The results obtained are shown in Figure 2 for the bandpass filter with $C_{1}=C_{2}=1000 \mathrm{pF}, I_{o 1}=I_{o 2}=1 \mu \mathrm{A}, 10 \mu \mathrm{A}$, $100 \mu \mathrm{A}, I_{o 3}=100 \mu \mathrm{A}, I_{o 4}=I_{o 5}=50 \mu \mathrm{A}$ and input current $I_{2}=1 \mu \mathrm{A}$, for the lowpass filter with $C_{1}=C_{2}=1000 \mathrm{pF}, I_{o 1}=I_{o 2}=1 \mu \mathrm{A}, 10 \mu \mathrm{A}$, $100 \mu \mathrm{A}, I_{o 3}=I_{o 4}=100 \mu \mathrm{A}, I_{o 5}=50 \mu \mathrm{A}$ and input current $I_{1}=1 \mu \mathrm{A}$, and for the highpass filter with $C_{1}=C_{2}=1000 \mathrm{pF}, I_{o 1}=I_{o 2}=1 \mu \mathrm{A}$, $10 \mu \mathrm{A}, 100 \mu \mathrm{A}, I_{o 3}=100 \mu \mathrm{A}=I_{o 4}=100 \mu \mathrm{A}, I_{o 5}=50 \mu \mathrm{A}$. and input current $I_{3}=1 \mu \mathrm{A}$. The results obtained by simulation are in fairly good agreement with the presented theory. 


\section{CONCLUSION}

A new universal second-order current-mode filter has been presented. The proposed filter uses the current-controlled current-conveyor and enjoys the following advantages:

a. current control of the parameters $\omega_{o}$ and $\omega_{o} / Q_{o}$ of the filters and the gains of the bandpass and the lowpass filters.

b. independent control of the parameter $\omega_{o}$ without disturbing the parameter $\omega_{o} / Q_{o}$ and the bandpass and the lowpass gains.

c. independent current control of the bandpass gain without disturbing the parameter $\omega_{o}$.

d. independent current control of the lowpass gain without disturbing the parameter $\omega_{o} / Q_{o}$.

e. low sensitivities of the parameters $\omega_{o}$ and $\omega_{o} / Q_{o}$.

\section{References}

[1] Chang, C.-M. and Chen, P. C. (1991). Universal active filter with current gain using OTAs, International Journal of Electronics, 71, 805-808.

[2] Nawrocki, R. and Klein, U. (1986). New OTA-capacitor realization of a universal biquad, Electronics Letters, 22, 50-51.

[3] Sanchez-Sinencio, E., Geiger, R. L. and Navarez-Lozano, H. (1988). Generation of continuous-time two integrator loop OTA filter structures, IEEE Transactions on Circuits and Systems, 35, 936-945.

[4] Wu, J. and Xie, C.-Y. (1933). New multifunction active filter using OTAs, International Journal of Electronics, 74, 235-239.

[5] Sun, Y. and Filder, J. K. (1993). Novel OTA-C realization of biquadratic transfer functions, International Journal of Electronics, 75, 333-340.

[6] Sun, Y. and Fidler, J. K. (1994). Versatile active biquad based on secondgeneration current conveyors, International Journal of Electronics, 76, 91-98.

[7] Chang, C.-M., Chien, C.-C. and Wang, H.-Y. (1994). Universal active current filter with three inputs using current conveyors-Part 2, International Journal of Electronics, 76, 87-89.

[8] Chang, C.-M., Chien, C.-C. and Wang, H.-Y. (1993). Universal active current filters using single second-generation current conveyor, Electronics Letters, 29, $1159-1160$.

[9] Chang, C.-M. (1991). Universal active current filters using single second-generation current conveyor, Electronics Letters, 27, 1614-1617.

[10] Chang, C.-M. (1991). Current mode allpass/notch and bandpass filter using single CCII, Electronics Letters, 27, 1812-1813.

[11] Chang, C.-M. (1993). Universal active current filter with single input and three outputs using CCIIs, Electronics Letters, 29, 1932-1933.

[12] Chang, C.-M. (1993). Novel universal current-mode filter with single input and three outputs using five current conveyors, Electronics Letters, 29, 2005-2006.

[13] Chang, C.-M. (1993). Current-mode lowpass, bandpass and highpass biquads using two CCIIs, Electronics Letters, 29, 2020-2021. 
[14] Roberts, G. W. and Sedra, A. S. (1992). A general class of current amplifier-based biquadratic filter circuits, IEEE Transactions on Circuits and Systems, 39, 257-263.

[15] Chang, C.-M. and Chen, P.-C. (1991). Universal active current filter with three inputs and one output using current conveyors, International Journal of Electronics, 71, 817-819.

[16] Wu, D.-S., Hwang, Y.-S., Liu, S.-I. and Wu, Y.-P. (1994). New multifunction filter using an inverting CCII and a voltage follower, Electronics Letters, 30, 551-552.

[17] Senani, R. (1992). New current-mode biquad filter, International Journal of Electronics, 73, 735-742.

[18] Chang, C.-M., Tu, S.-H. and Yieh, P.-W. (1994). Synthesis of analog signal filtering circuits using second-generation current conveyors, Proceedings National Science Council, ROC(A), 18, 421-427.

[19] Horng, J.-W., Lee, M.-H. and Hou, C.-L. (1995). Universal active filter using four OTAs and one CCII, International Journal of Electronics, 78, 903-906.

[20] Fabre, A., Saaid, O., Wiest, F. and Boucheron, C. (1996). High frequency applications based on a new current controlled conveyor, IEEE Transactions on Circuits and Systems-I: Fundamental Theory and Applications, 43, 82-91.

[21] Fabre, A., Saaid, O., Wiest, F. and Boucheron, C. (1995). Current controlled bandpass filter based on translinear conveyors, Electronics Letters, 31, 1727-1728. 

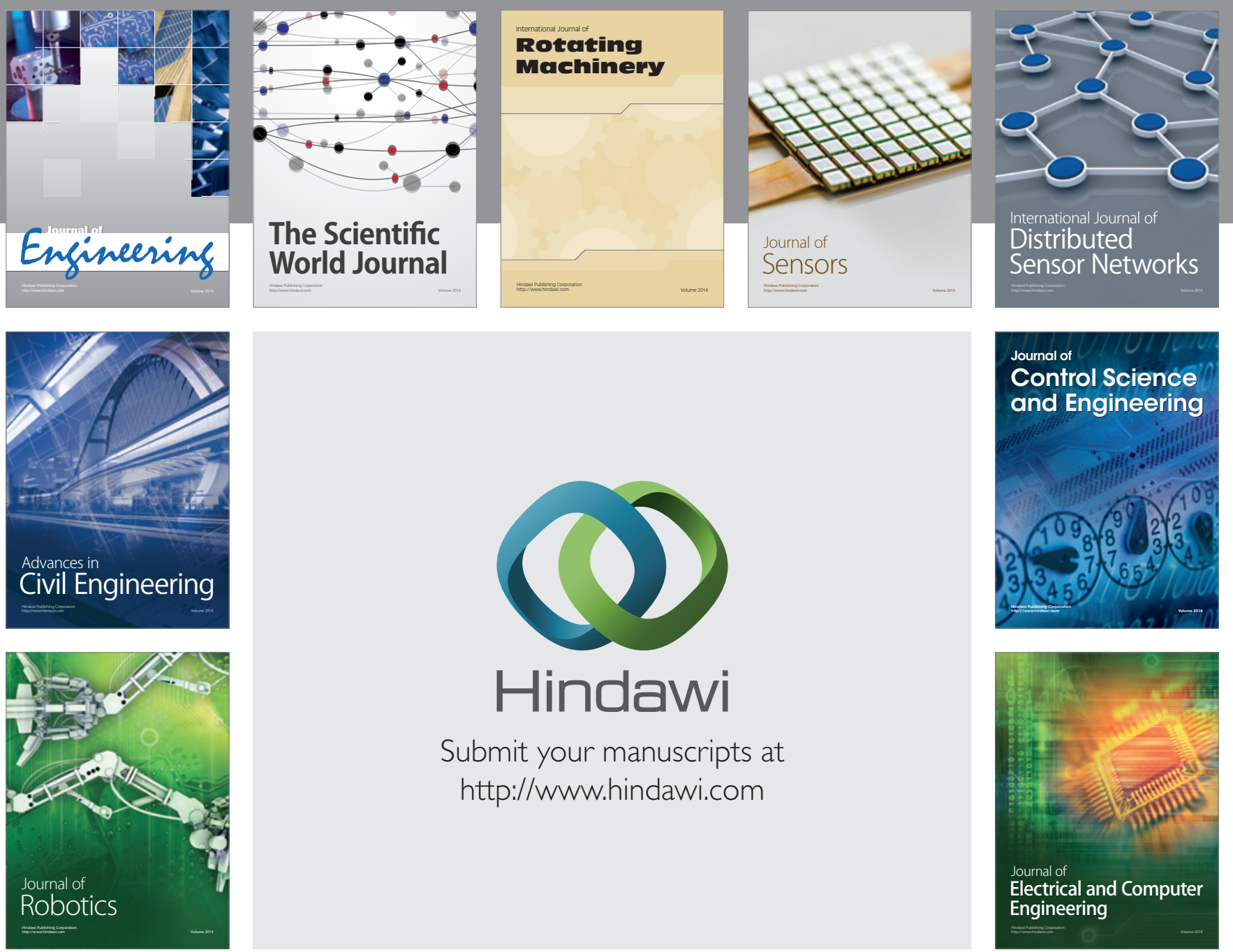

Submit your manuscripts at

http://www.hindawi.com
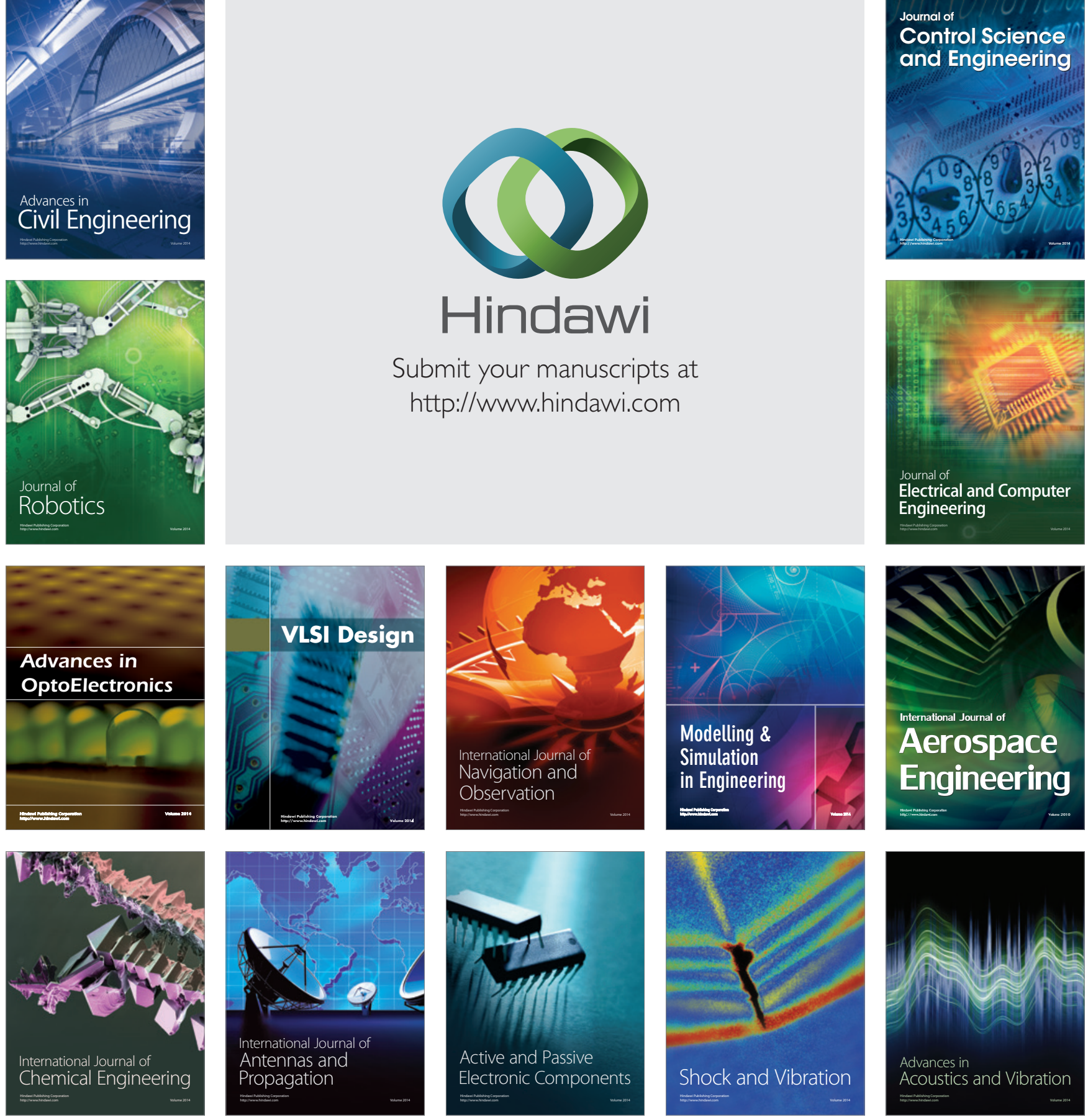